\title{
Thiel Cadaveric Nerve Tissue: A Model for Microsurgical Simulation
}

\author{
Andrei Odobescu ${ }^{1}$ Sami P. Moubayed ${ }^{2}$ Michel Alain Danino ${ }^{1}$ \\ ${ }^{1}$ Department of Plastic and Reconstructive Surgery Service, Université \\ de Montréal Hospital Center, Montreal, Canada \\ 2 Department of Otolaryngology-Head and Neck Surgery Service, \\ Université de Montréal Hospital Center, Montreal, Canada \\ J Brachial Plex Peripher Nerve Inj 2016;11:e18-e20.
}

Address for correspondence Michel Alain Danino, MD, PhD, NotreDame Hospital, 1560 rue Sherbrooke Est, Montreal, Quebec, H2L 4M1, Canada (e-mail: michel.alain.danino@umontreal.ca).

Peripheral nerve research as well as nerve repair simulation has relied heavily on the rat animal model, more specifically on the rat sciatic nerve. ${ }^{1}$ As the use of animals in experiments and training has received much criticism from animal rights activists and society at large, the field of surgical simulation is currently emerging. In microsurgery, high-fidelity Silastic models, animal parts such as chicken thighs or wings, and cadaveric specimens have been used. Based on the available experience with Thiel embalmed cadaveric tissue in simulation, ${ }^{2-4}$ we experimented with Thiel embalmed peripheral nerves for the purpose of microsurgical skill training.

We used median, ulnar, and tibial nerves from cadavers that had been used for anatomic and surgical training and had not touched the peripheral nerve tissue. The donors had previously consented to tissue utilization in postmortem research. The tissues originated from cadavers prepared with the embalming method described by Thiel. ${ }^{5}$ This technique preserves texture, volume, color, and shape of the body as perfect as possible, with the advantage of avoiding decay observed with fresh cadaveric specimens. There is no shrinking or soaking of the soft tissues. Thirteen nerve sections measuring $5 \mathrm{~cm}$ each were prepared on a foam board. Needles $(25 \mathrm{G}$ ) are used to fix the nerves to the foam board. A blue background was used for the exercise, as it improves contrast. An operating microscope (Opmi Pico, Carl Zeiss, Oberkochen, German) at $10 \times$ magnification was used for all microneurorrhaphies.

Under magnification, the nerves were crushed in the midsection to simulate an injured nerve. The participants transected the nerve using a 15-blade scalpel, and trimmed the damaged nerve tissue. The two ends were inspected for the fascicular architecture and oriented appropriately for the repair. The epineurium was then gently reflected back and the proud fascicles trimmed. Nylon 8-0 sutures were used to perform a simple epineural repair, starting with the 0 - and 180-degree orientation sutures and then filling in the required sutures to obtain a well-oriented microneurorrhaphy.

received

January 3, 2016

accepted

February 8, 2016
DOI http://dx.doi.org/

$10.1055 / \mathrm{s}-0036-1580626$. ISSN $1749-7221$.
Under magnification of the operative microscope, we found the Thiel nerve tissue to show a slight gray-brown discoloration with an epineural layer that was hydrophilic, giving the impression of edematous tissue (-Fig. 1). This thicker-than-normal epineural layer, however, offers adequate support for manipulation. Unfortunately, the cadaveric nature of the model precludes the use of the vasa nervorum, which are not visible, for adequate orientation of the nerve. Upon transection of the nerve, it can be observed that the fascicles are well preserved and bound by firm endoneurium and perineurium which have not undergone the same edema as the epineurium ( - Fig. 1). Despite there being no immediate herniation of nerve fascicles upon transection, the fascicles have a tendency to be more hygroscopic, and by the end of the neurorrhaphy, one can observe some protrusion of fascicles in between suture. The fascicular pattern is easily identifiable and permits good alignment of the nerve before suturing.

Thirteen volunteer plastic surgery, otolaryngology, and orthopedics residents utilized the model once each, and filled out a postsimulation survey. The results were graded on a five-point Likert scale (strongly agree, disagree, neither agree nor disagree, agree, strongly agree). A question regarding the frequency participants would use the laboratory with answers graded in five categories was also asked. The contents of the postsimulation survey are presented in - Table 1. Descriptive statistics are presented for the results of the survey questions. All participants (100.0\%) agreed that they would use the module at least twice a year, with $53.9 \%$ (seven residents) stating they would use it more than once a month, $38.5 \%$ (five residents) once a month, and $7.7 \%$ (one resident) twice a year.

The rapid development of microsurgery over the last three decades has been echoed by the development of several simulation models for the teaching and honing of microsurgical skills. Free flaps are routine procedures in most plastic surgery centers, and residents have ample opportunity to participate and perform in these procedures. As a

Copyright $\odot 2016$ Georg Thieme Verlag KG Stuttgart · New York
License terms

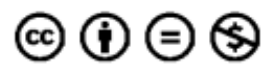




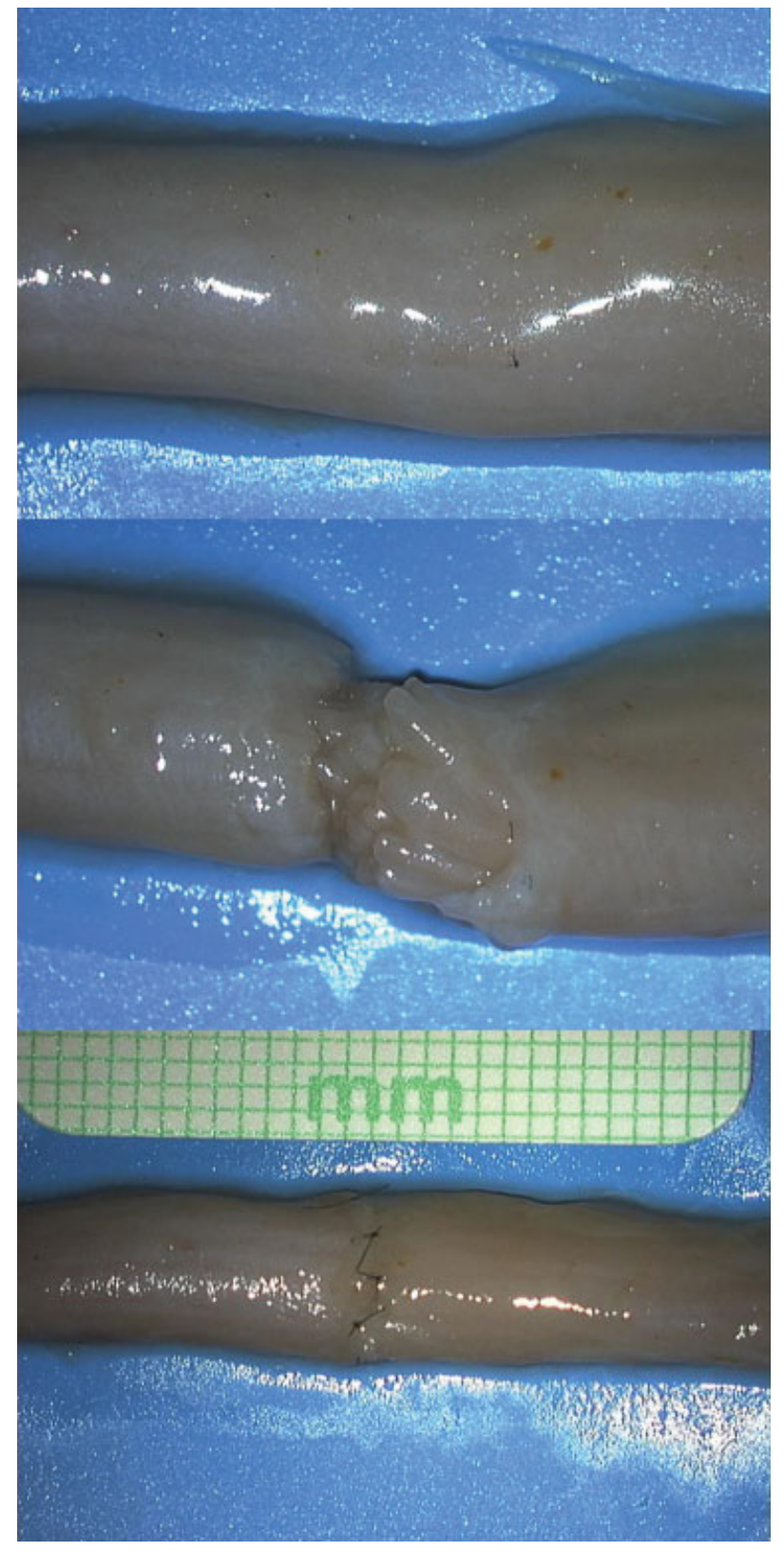

Fig. 1 Thiel embalmed nerve for nerve repair simulation. Upper panel: intact nerve before transection showing a hygroscopic epineurium. Middle panel: transected nerve showing the architecture of the fascicles. Lower panel: completed nerve repair.

consequence, microsurgical laboratories have been established, both on artificial, ex vivo animal and live animal models. Few of these models have been validated. According to a systematic review by Dumestre and colleagues, out of the 238 articles published in the literature pertaining to microsurgical training models, 9 have been adequately validated. These include several artificial pads or cards, chicken or turkey legs or wings, and the current gold standard for microvascular simulation, the rat femoral artery. ${ }^{6}$ Peripheral nerve surgery simulation is lagging behind. One of the only reports of nonanimal models for nerve microsurgical simulation comes from Senturk and colleagues, who published a model consisting of rubber threads. ${ }^{7}$ The argument for such a

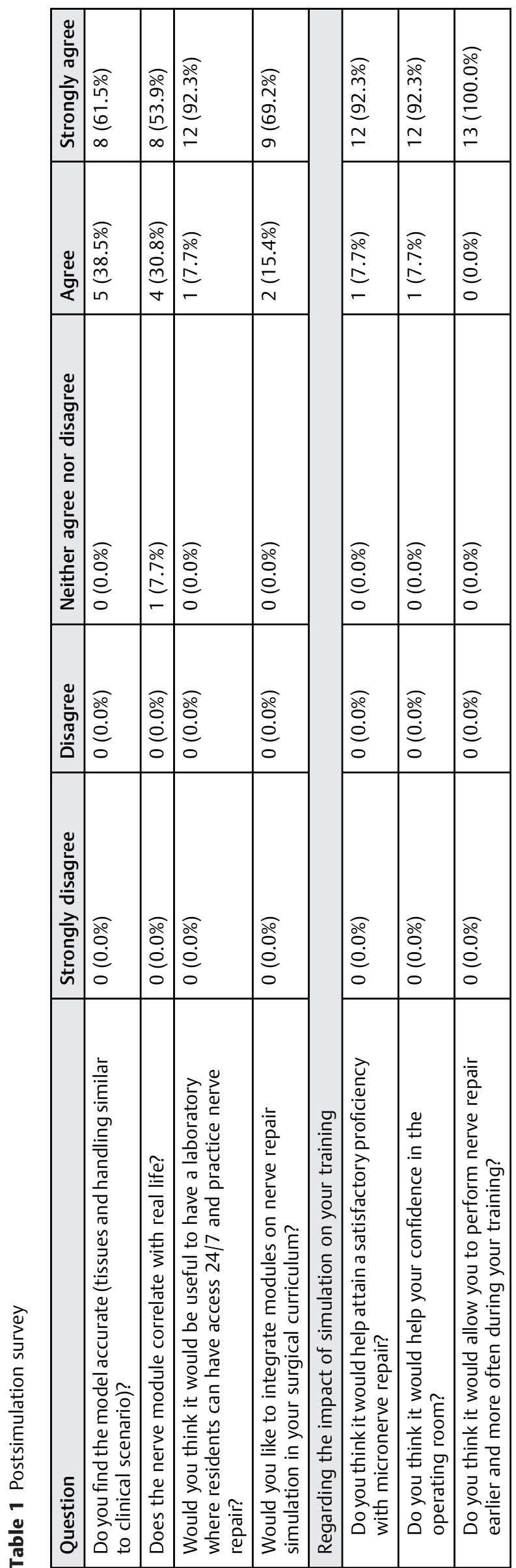


model is the low cost and relative similarity of the architecture, with a bundle of elastic fibers wrapped up by a mesh. Yet the physical fidelity of such a model is low. The technical difficulty of nerve repairs is often underestimated, since there is no immediate failure as seen in microvascular surgery; a poor result is often seen months later and there are often multiple causes for the outcome, including the initial trauma and scarring, yet the effect of poor technique and iatrogenic trauma during repair should not be underestimated. It is for these reasons that simulation in peripheral nerve surgery is valuable.

We believe the Thiel peripheral nerve model to be a high physical and functional fidelity model that permits trainees to become accustomed with the basic principles of microsurgical nerve repair. In addition, it allows the trainee to perform multiple repairs of one median nerve, maximizing the educational benefit per cadaveric specimen. Thiel embalmed nerves can be harvested from cadavers that have already been used for other educational exercises, to ethically maximize their use, and the specimens can be stored for extended periods of time in the appropriate Thiel solution (in our experience up to 2 years). The Thiel nerve model will need to be formally validated in future studies in terms of surgical skill improvement.

\section{Grant Sponsor}

None.

\section{References}

1 Tamai S, Usui M, Yoshizu T. Experimental and Clinical Reconstructive Microsurgery. Tokyo: Springer; 2003

2 Hassan S, Eisma R, Harry LE. Surgical training of anastomotic technique using Thiel cadavers. J Plast Reconstr Aesthet Surg 2014;67(10):e250-e251

3 Hassan S, Eisma R, Malhas A, Soames R, Harry L. Surgical simulation flexor tendon repair using Thiel cadavers: a comparison with formalin embalmed cadavers and porcine models. J Hand Surg Eur Vol 2015;40(3):246-249

4 Wolff KD, Kesting M, Mücke T, Rau A, Hölzle F. Thiel embalming technique: a valuable method for microvascular exercise and teaching of flap raising. Microsurgery 2008;28(4): 273-278

5 Thiel W. The preservation of the whole corpse with natural color [in German]. Ann Anat 1992;174(3):185-195

6 Dumestre D, Yeung JK, Temple-Oberle C. Evidence-based microsurgical skill-acquisition series part 1: validated microsurgical models-a systematic review. J Surg Educ 2014;71(3): 329-338

7 Senturk S, Tosun Z, Ozkan A. Microsurgical training model for nerve repair. J Reconstr Microsurg 2005;21(7):491-492 